\title{
Evaluation of efficacy and regenerative potential of Wharton's jelly and bone marrow derived mesenchymal stem cells in diabetic rats
}

\author{
Chitra Som', Neelam K Venkataramana' \\ 1 Advanced Neurosciences Allies Indira Nagar Bangalore, India \\ Som Ch, Venkataramana N. K, Evaluation of efficacy and regenerative potential of Wharton's jelly and bone marrow derived mesenchymal \\ stem cells in diabetic rats. J Pre-Clin Clin Res.2018; 12(1): 30-35. doi: 10.26444/jpccr/87143
}

\begin{abstract}
Introduction. Diabetes is a chronic metabolic disorder that to-date has no definitive cure. The main challenge for successful therapy to treat diabetes lies in producing functional $\beta$-cells and overcoming the autoimmune response. Currently; regenerative medicine using Mesenchymal Stem Cells (MSCs) offers promising treatment.

Objective. Comparison of the antidiabetic effect of bone marrow MSCs with Wharton's jelly MSCs in streptozotocin-induced diabetes (T1D) in rat models.

Materials and Method. Wharton's jelly derived from human umbilical cords and bone marrows aspirated from the posterior iliac crest were used for the isolation of WJ-MSCs and BM-MSCs, respectively. MSCs were cultured and cells from passage 6 were used for the study. Experimental animals were induced with T1D using multiple low dose streptozotocin (MLDSTZ). Experimental mice were divided into 6 groups $(n=10)$; Groups 1 and 2 were diabetic and normal controls, Groups 3 and 4 were Diabetic mice treated with WJ-MSCs and BM-MSCs, and Groups 5 and 6 were diabetic mice given insulin and glybenclamide treatments, respectively.

Results. Low blood glucose levels, increased serum insulin levels, and significant pancreatic regeneration were observed in WJ- MSCs treated diabetic group. This was accompanied by improvement of the histopathological changes. The results were not promising in the BM-MSCs groups which had no noticeable changes.

Conclusion. The source of MSCs from where it is procured is one of the main factors that influence the differentiation potential of MSCs into insulin producing cells. WJ-MSCs are better antidiabetic agents than BM-MSCs, and are par to the standard diabetic drugs, coupled with potential to initiate pancreatic regeneration.
\end{abstract}

\section{Key words}

diabetes, Mesenchymal stem cells, Wharton's jelly, bone marrow

\section{INTRODUCTION}

Diabetes is one of the most conceded endocrine metabolic disorders designated by chronic hyperglycaemia. It occurs either when the pancreas does not produce enough insulin or when the body cannot effectively use insulin. It is associated with numerous health complications, including increased risk for heart disease, stroke, nephropathy, blindness, and amputations. [1]. Type 1 diabetes (T1D) is characterized by an initial autoimmune targeting of the islets that results in a loss of self-tolerance and inflammatory destruction of the insulin producing $\beta$-cells [2]. Exposure to a trigger initiates the autoimmune damage which is followed by insulitis, characterized by invasion of islets by mononuclear cells leading to the death of $\beta$-cells [3]. In type 2 diabetes, inflammationinduced inhibition of the insulin signalling pathway leads to insulin resistance and triggers disease onset [4]. In addition to hyperglycaemia, diabetes is characterized by specific (microvascular) or nonspecific (cardiovascular) complications. Diabetes management includes treating cardiovascular risk factors, such as hypertension and dyslipidemia, often to more stringent goals than those for patients who do not have diabetes. Despite all the currently available drugs, the control

Address for correspondence: Chitra Som, Advanced Neurosciences Allies\ nIndiranagar \nBangalore, India, 9th 'A' Main, off CMH Road, Indira Nagar III Stage, Hoysala Nagar, Karnataka 560038 Bengaluru, India

E-mail: chitramarine@yahoo.com

Received: 27 November 2017; accepted: 9 February 2018 of diabetes is still a challenge since the overall management of diabetes itself and its associated complications is not satisfactorily addressed by the current treatment strategies.

To-date, there is no definitive cure for diabetes because insulin injection does not mimic the precise regulation of $\beta$-cells on glucose homeostasis, and does not prevent development of associated complications [4]. Attributed to this, diabetes falls into the category of disabling disease that can severely impact the quality of life of those who are afflicted. Currently, the therapeutic potential of stem cells is being sought globally, an area that has gained great interest is the tremendous potential of stem cells in diabetes research. The prevailing inclination is attributed to the regenerative capabilities and immunomodulatory properties of mesenchymal stem cells. In the purview of diabetes, MSCs have been used to generate insulin-producing cells [5], curb autoimmunity [6], enhance islet engraftment [7] and to treat diabetic ulcers and limb ischemia [8]. Furthermore, MSC inoculum helped improve metabolic control in experimental models of type 2 diabetes (T2D).

However, the therapeutic value of MSCs for enfeebling the autoimmune disorder type 1 diabetes is logically sound although MSC treatment of this particular disease remains largely unexplored. Nevertheless, mesenchymal stem cells (MSC) from different tissue sources share many characteristics and generally fulfill accepted criteria for MSCs (plastic adherence, specific surface marker expression, and ability to differentiate into mesenchymal tissues), progress is 
being made in understanding that they can be differentiated at the level of cytokine production and gene expression profiles. Their ability to individualize into different tissues, including endodermal and ectodermal lineages, also varies according to tissue origin [9].

MSCs were primarily isolated from bone marrow (BM) and is still considered as the archetype for MSC applications [10]. Nonetheless the BM has limitations as a source of MSCs, primarily due to the painful isolation procedure and also due to the relapse in MSC characteristics with donor's age [11]. This demands an increased interest in identifying alternative sources for MSCs. Mesenchymal stem cells obtained from Wharton's Jelly (WJ) from umbilical cords (UC) have gained much attention over the last few years due to their easy isolation from cord tissue which is discarded after birth. Besides WJ -derived MSCs represent a more primitive population than their adult counterparts, opening new perspectives for cell-based therapies [12]. Hence, this study was designed to compare the antidiabetic effect of BM-MSCs and WJ-MSCs on streptozotocin induced T1D in a rat model, as a step towards providing scientific knowledge to escalate the therapeutic potential to clinical levels.

Ethical approval of the Institutional Research Committee was obtained to perform the study.

\section{MATERIALS AND METHOD}

Experimental animals. This study was conducted on wistar rats, weighing 250-300 grams at the beginning of the study. The animals were acclimatized to their new environment for one week before the start of the experiment. They were housed in a cage, with free access to food and water. Animals were anaesthetized before scarification.

Study design. All experimental animals were divided into 6 groups ( $\mathrm{n}=10)$ : 1) Non Diabetic Control group; 2) Diabetic Control group: induced with diabetes, 3)WJ MSCs treated diabetic group: after 72 hours of diabetes induction animals were treated twice with intravenous injection of $1 \times 10^{6}$ cells $/$ rat, 24 hours apart, without immunosuppression, 4) BM-MSCs treated diabetic group: received the same dose of MSCs, 5) Diabetic rats given insulin therapy from $72 \mathrm{hrs}$ after induction of diabetes, and 6i) diabetic rats given glybenclamide from $72 \mathrm{hrs}$ after induction of diabetes. Duration of the study was 12 weeks.

Induction of diabetes. For the diabetic groups, T1D was induced using daily I.P. injection of MLD-STZ in a dose of $40 \mathrm{mg} / \mathrm{Kg}$ [13], dissolved in citrate buffer $\mathrm{pH} \mathrm{4.5,} \mathrm{for} 5$ consecutive days. Blood glucose levels were measured before the induction of diabetes, after the induction, and then every 3 days for 30 days, using a One-Touch Glucometer (MDSS $\mathrm{GmbH}$, Germany). The measurement was performed by puncturing the tail vein to obtain a drop of blood. Animals were considered diabetic when random blood sugar was $\geq 250 \mathrm{mg} / \mathrm{dl}$.

\section{PREPARATION OF MSCS}

Isolation and culture of WJ-MSCs. Human umbilical cords from both genders were collected from full-term births after either Caesarean section or normal vaginal delivery with informed consent, using the guidelines approved by the Institutional Committee for Stem Cell Research and Therapy (ICSCRT) and Institutional Ethics Committee (IEC) at the Manipal Hospital, Bangalore, India. MSCs from WJ of umbilical cord were isolated as previously described [14]. After the enzymatic treatment, cells were suspended in 10\% FBS (Hyclone, Victoria, Australia) and knockout Dulbecco's modified Eagle's medium (DMEM-KO) (Invitrogen, CA, USA) and plated on tissue culture plastic plates (Falcon, Becton, Dickinson \& Co., NJ, USA). WJ-MSCs were cultured in DMEM-KO with $4,500 \mathrm{mg} / \mathrm{ml}$ glucose and 2mM L-glutamine (Invitrogen), supplemented with 10\% FBS (Hyclone, Cat. No.SH30084.03, Lot No.GQM0049). The culture was maintained at $37^{\circ} \mathrm{C}$ in a humidified atmosphere containing $95 \%$ air and $5 \% \mathrm{CO}_{2}$. All cultures were plated at a density of 5,000 cells/cm 2 and passaged when they reached $70-80 \%$ confluence.

Isolation and culture of BM-Mesenchymal stem cells. BMMSCs were isolated from healthy, screened donors between the ages of 18-30 years, with informed consent. $60 \mathrm{ml}$ of bone marrow was aspirated aseptically under local anesthesia from iliac crest of the healthy screened donors. The samples were transported appropriately to the processing laboratory, a class 1000 cGMP facility, and all sample processing was performed in a class 100 biosafety cabinet. Boney spicules and particles were removed using a cell strainer and further diluted with DMEM-KO, centrifuged at $1,800 \mathrm{rpm}$ for $10 \mathrm{~min}$ at $20^{\circ} \mathrm{C}$. The cells were resuspended with DMEM-KO and gently layered onto a density gradient solution (Lymphoprep, Axis Shield PoC AS, Oslo, Norway) to obtain an enriched mononuclear fraction. This was washed with DMEM-KO and centrifuged to collect the cells. The cells obtained were resuspended and plated in MSC complete culture medium consisting of Dulbecco's modified Eagle Medium (DMEM-KO) 10\% foetal bovine serum (FBS) from preselected lots and glutaMAX, as described elsewhere [14]. The culture was maintained at $37^{\circ} \mathrm{C}$ in a humidified atmosphere containing 95\% air and 5\% CO2 and subcultured prior to confluency.

Subculturing and expansion. Once $80 \%$ confluent the cells were dissociated with $0.25 \%$ trypsin/0.53mM EDTA (Invitrogen) they were further upscaled and expanded in order to provide the required number of cells for the study. Briefly, trypsinized cells were reseeded at a density of 1,000 cells per $\mathrm{cm}^{2}$ in cell stacks (Corning) up to passage 6. After 14 days in culture, the cells reached $80 \%$ confluency and used in the study.

Quality control testing. MSCs were tested for quality control parameters, e.g. Mycoplasma, Endotoxin, sterility and cell surface markers, such as CD73, CD90, CD105, CD166, CD34, and CD45 markers using flow cytometry. They should be more than $80 \%$ positive for CD73, CD90, CD166, and CD105 but negative $[<10 \%]$ for CD34 and CD45.

Characterization of Mesenchymal stem cells - Immunophenotype. Immunophenotyping of the cultured MSCs was performed using flow cytometry to identify the presence of specific cell-surface antigens, as described earlier. MSCs were dissociated with $0.25 \%$ trypsin- EDTA and resuspended in wash buffer. Cell viability was measured by flow cytometry 
using 7-amino actinomycin D (7-AAD). $200 \mu \mathrm{L}$ cell suspension were incubated in the dark for $30 \mathrm{~min}$ at $4^{\circ} \mathrm{C}$ with saturating concentrations of phycoerythrin PE-conjugated antibodies. Appropriate isotype-matched controls were used to set the instrument parameters. After incubation, cells were washed 3 times with wash buffer and resuspended in $0.5 \mathrm{~mL}$ wash buffer for analysis. Flow cytometry was performed on a $5 \mathrm{HT}$ Guava instrument. Cells were identified by light scatter for 1,0000 gated events and analyzed. The following markers were analyzed: CD34-PE, CD45-PE, CD73-PE, CD105-PE, CD166$\mathrm{PE}$, and CD90-PE (BD Pharmingen, San Diego, CA,USA).

Histological assessment of pancreas sample by Heamatoxylin Eosin (H/E) staining. At the end of the study period, all the rats of different groups were sacrificed using mild anaesthesia. The liver, kidney and pancreas tissues were fixed in neutral formalin solution for $48 \mathrm{~h}$, dehydrated by passing through graded series of alcohol embedded in paraffin blocks. Hematoxylin and Eosin (H and $\mathrm{E})$ stained paraffin sections, $4 \mu \mathrm{m}$ thick, were prepared.

Statistical analysis. Data were expressed as mean \pm SEM. Comparisons among groups were carried out using one-way ANOVA. All $\mathrm{p}$ values reported are 2 -tailed and $\mathrm{p}<0.05$ was considered significant.

\section{RESULTS}

Morphology. Morphological evaluation of the cells revealed shiny, spindle-shaped, fibroblast- shaped cells with clear cytoplasm, with no granulations detected.

Imunophenotyping. The cells, BM and WJ MSCs, were characterized for $\mathrm{CD}$ markers as per the international criteria. The cells were found to be negative for CD34, CD45, HLA-DR and positive for CD73, CD90 and CD44.

Clinical observations. At the beginning of the experiment, all animals weighed between 250 - 300 grams, which gradually increased in the control group but progressively decreased in the diabetic groups, to range between $180-200$ grams at the 30th day of the study. Feed/water intake and urination were increased in diabetic animals with progressive decrease in body weight and animal activity. In the groups treated with WJ MSCs, Insulin and Glybenclamide all the previous signs observed in the diabetic group gradually improved, starting from week 1 . The results were statistically significant. However, the results were not significantly comparable in group treated with BM-MSCs.

Blood glucose level measurement. In the control group, blood glucose levels were normal (100-120 mg/dl) throughout the experiment, whereas in the diabetic groups they were 250-500 mg/dl until the end of the experiment. In the groups treated with WJ-MSCs (Graph 1), insulin (Graph 2) and Glybenclamide (Graph 3), the animals were diabetic during the first week after induction, after which the blood glucose levels gradually decreased, starting from week one and became normal by week 6 . These results were statistically significant when compared to the diabetic groups. There was no notable decrease in the blood glucose level of animals in the group treated with BM-MScs (Graph 4).

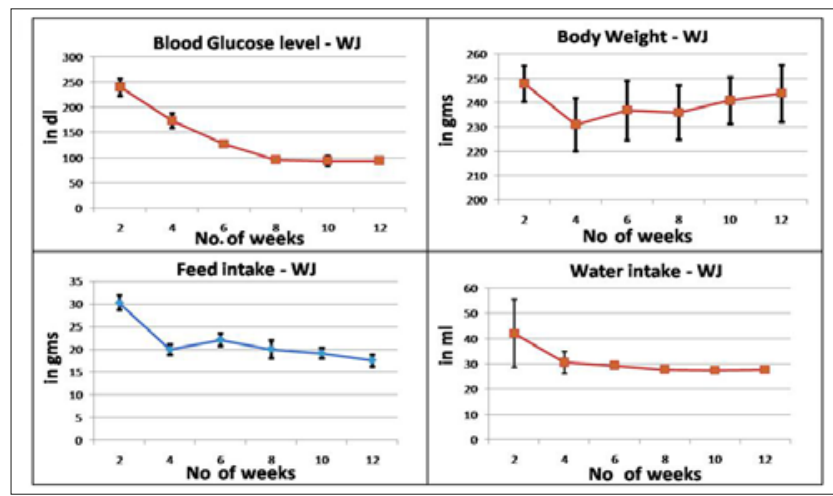

Graph 1. Diabetic parameters in STZ rats after Wharton's jelly MSCs transplantation

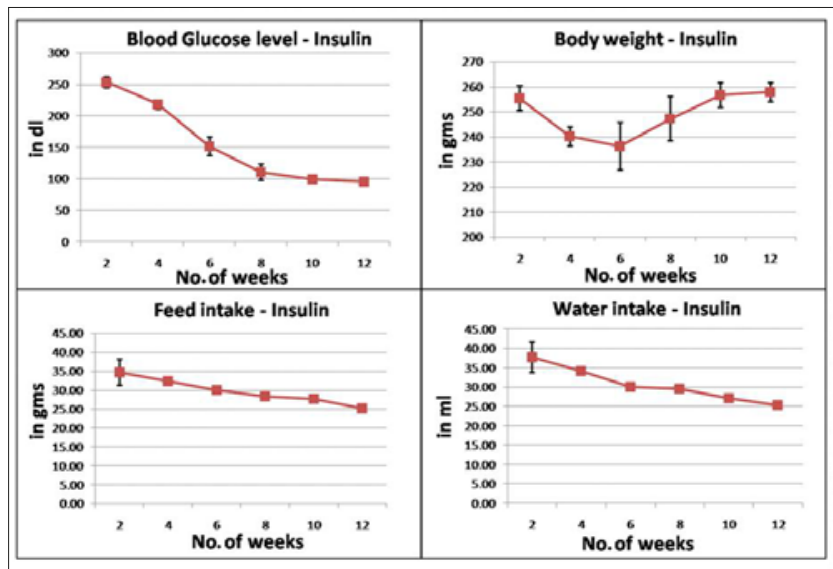

Graph 2. Diabetic parameters in STZ rats after Insulin therapy

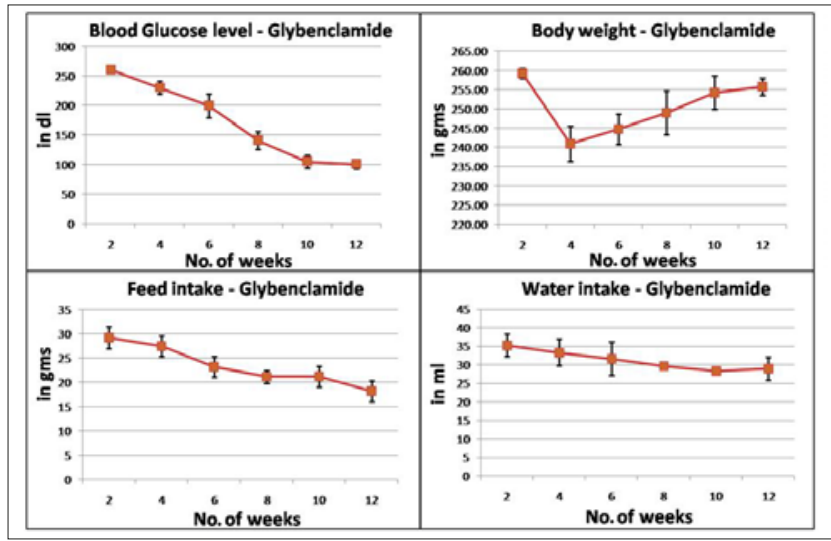

Graph 3. Diabetic parameters in STZ rats after Glybenclamide therapy

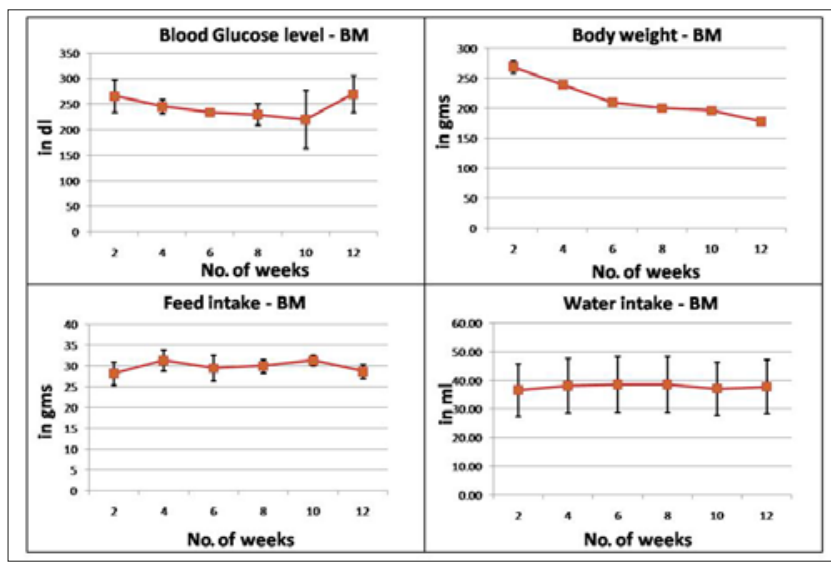

Graph 4. Diabetic parameters in STZ rats after bone marrow MSCs transplantation 
Serum insulin levels. There was an increase in blood sugar and a decrease in insulin levels in the first week. Later, there was a gradual increase in insulin levels in all groups. However, at the end of the study period, the WJ transplanted group showed higher insulin levels compared to the other treated groups.

Histological assessment. A normal pancreas section shows well-arranged cells maintaining normal morphology of acini and the islet cells. The STZ-treated diabetic groups, however, showed pancreas with necrotic acini and grossly shrunken and distorted islet cells (Fig. 1A, 1B). The WJ MSC's treated diabetic group showed signs of regeneration with noticeable changes involving near normal acini and pancreatic islets. The BM MSC treated group showed no signs of regeneration of pancreas. Near normal acini with grossly distorted islet cells were observed (Fig. 2A, 2B). The diabetic groups treated with insulin and Glybenclamide also showed no signs of pancreatic regeneration. Near normal acini with grossly distorted islet cells were commonly observance in both the groups (Fig. 3A, 3B).

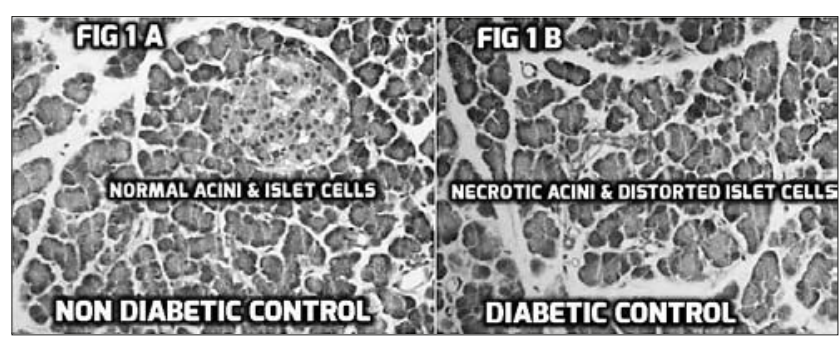

Figure 1A, 1B. Non-diabetic control and diabetic control

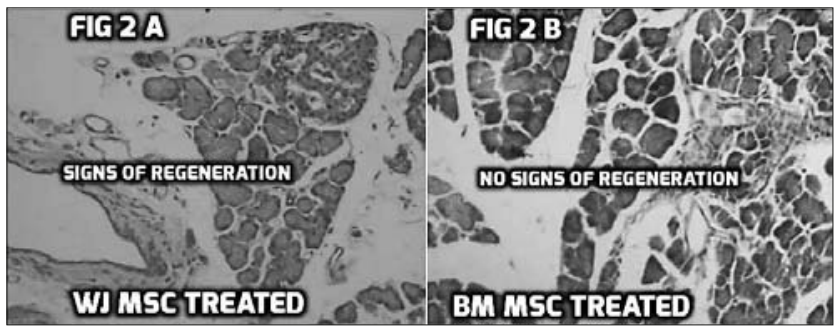

Figure 2A, 2B. WJ MSC treated and BM MSC treated

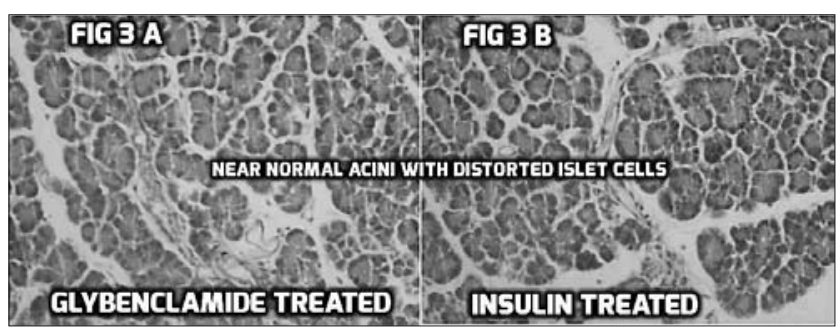

Figure 3A, 3B. Glybenclamide treated and insulin treated

\section{DISCUSSION}

Diabetes is considered the most burdensome diseases for patients, their families, and the health care system. The complications developing from diabetes mellitus have significant health, economic and social impacts on individuals. The complications that are specific to diabetes include retinopathy, nephropathy, and neuropathy. Controlled clinical trials involving patients with type 1 diabetes and those with type 2 diabetes have conclusively demonstrated that intensive diabetes therapy aimed at lowering glycaemic levels reduces the risk of cardiomyopathy. Patients with all forms of diabetes of sufficient duration, including insulindependent diabetes mellitus (IDDM) and non-insulindependent diabetes mellitus (NIDDM), are vulnerable to these complications which cause serious morbidity.

Diabetics have had ongoing issues with different drugs to treat their condition. The most prominent of the oral drugs in diabetes treatment are Pioglitazone, Rosiglitazone, Sulfonylureas and Metformin, but the long-term use of these drugs are reported to result in complications, including heart failure, heart-related risks, kidney failure, and even cancer [15]. Intensive glucose-lowering therapy with insulin intermittently results in increased risks of mild and severe hypoglycaemic episodes requiring medical assistance. The prevalence of diabetes is anticipated to rise in the coming years [15], therefore a therapeutic strategy aimed at repairing insulin- producing cells and preventing the autoimmune destruction of vestige and newly-generated cells is highly desirable.

Prior reports highlight the role of the immune mediated inflammatory process in causing loss of pancreatic beta cells in both type 1 and type 2 diabetes. Type 1 diabetes (T1D) is characterized by an initial autoimmune targeting of the islets. Its targeting is due to a shortcoming in the immune system resulting in a loss of self-tolerance and inflammatory destruction of the insulin producing $\beta$-cells [2]. The pathogenesis of autoimmune type 1 diabetes becomes initiated by the $\beta$-cell autoantigens processed by macrophages, dendritic cells, or B cells in the pancreatic islets, and presented to autoreactive CD4+ T cells in the peripheral lymphoid system. The autoreactive CD4+ T cells are activated and secrete cytokines, which can activate b cell-specific cytotoxic CD8+ T cells. The activated T cells are recruited to the pancreatic islets and produce cytokines, which further activate macrophages and other $\mathrm{T}$ cells, contributing to the destruction of $\beta$-cell [16]. In type 2 diabetes, it is reported that inflammation-induced inhibition of the insulin signalling pathway leads to insulin resistance and contributes to the disease onset [17]. MSCs represent a propitious tool for this due to their trans-differentiation potential, as well as to their immunomodulatory properties [16].They suppress T-cell proliferation and also significantly reduce the enunciation of certain activation markers on stimulated lymphocytes [17]. At the preclinical level, prior reports have proved that systemic administration of MSCs results in a recovery of pancreatic islets, increases blood insulin secretion, and corrects hyperglycaemia $[6,18]$

In the presented study, multiple low dose streptozotocin were used for induction of T1D. It has been stated that STZ diabetic animals may exhibit most of the diabetic complications mediated through oxidative stress [13]. This drug causes damage to $\beta$-cells of the pancreas, mainly through two different pathways; the preliminary mechanism induces direct toxicity to the cells through production of nitric oxide and oxidative damage to the cells. The secondary mechanism is T-cell mediated immunological process which initiates the pathophysiology of diabetes.

Glibenclamide is an orally administered standard drug that stimulates insulin secretion from beta cells of the islets of Langerhans, thereby reducing the glucose concentration. Hence, it is made use of in many studies as a standard 
antidiabetic drug in STZ- induced diabetes to compare the antidiabetic properties of a variety of hypoglycaemic compounds. Intraperitoneal administration of insulin as a standard antidiabetic drug enhances glucose uptake across the cell membrane by ATP-dependent translocation of glucose transporter GLUT4 to the plasma membrane, and is the gold standard to compare the antidiabetic properties of other compounds. In the presented study, both, Glibenclamide and insulin were used as the standard diabetic therapeutics for the hypoglycaemic activity comparison of mesenchymal stem cells. The current study corroborates prior findings that mesenchymal stem cells are ideal candidates for diabetes treatment, since the presented study groups showed a comparable activity with the Glibenclamide and insulin treated groups. However, the effects were more pronounced in the WJ- MSC treated group. The obtained results show that one week after administration and up to the end of the follow-up period, WJ-MSC treated diabetic rats corrected hyperglycaemia, and were observed with increased blood insulin levels.

It is evidenced that MSCs from foetal sources, when compared to adult sources, creates a large population of immature unprimed highly functional regulatory $\mathrm{T}$ lymphocytes [19]. These regulatory $\mathrm{T}$ cells are reported to help decrease the inflammatory cytokine response that instigates diabetes. Even the presented results are in favour of WJ-MCs obtained from foetal sources as more viable in abating diabetes than BM- MSCs.

It is apparent from the different data sources that MSCs, although they may fulfill the common criteria of plastic adherence and fibroblast-like morphology, multilineage differentiation capacity, expression of surface markers such as CD73, CD90, and CD105, and lack of lineage-specific markers, such as CD34, CD14, CD45, they still show disparity in their bioactivity profiles [9]. These disparities mainly arise from the tissue source from which the cells are procured, and also with the cell culture passages $[11,20]$. One of the main factors that seem to affect the differentiation potential of MSCs into insulin producing cells include the source of MSCs, which seems to play an important role in their response to differentiation factors. It is concluded from earlier reports that MSC from foetal sources can undergo more cell divisions before they reach senescence than MSC from adult tissue, such as bone marrow [21]. These observations are in line with the presented results which show a better recovery of the WJ-MSCs treated groups than the BM-MSCs treated groups.

Histological observations of the pancreas in this study revealed signs of regeneration with noticeable changes involving near normal acini and islets in the WJ MSC's treated diabetic group. However, the BM MSC's treated group showed no signs of regeneration. Because of the foetal origin of WJ-MSCs along with expression of embryonic and endoderm lineage markers, they are more similar to embryonic stem cells than the BM-MSCs [21-22]. This resemblance to embryonic cells should have rendered WJMSCs a more superior differentiation into insulin producing cells, as evidenced by this study.

It was also observed that the biological and immunophenotypical characteristics of cultured BM-MSC showed pronounced alterations with increasing numbers of passage of culture. Earlier studies demonstrate that the increase in donor age contributed to the decreased proliferation and differentiation capacity of BMSCs in culture [23]. A previous study on the immune properties of different mesenchymal stem cells also reported that around passage 6, BM-MSCs reach cellular senescence; but on the other hand, umbilical cord derived MSCs at this passage are far from showing any signs of ageing [24]. The MSCs made use of in the current study came from passage 6 , and the results obtained agree with the previous reports that BM- MSCs from passage 6 are less viable when compared to WJ-MSCs from the same passage.

The results obtained in this study show that WJ-MSCs and BM-MSCs do not behave in the same way, and depending on the source of the MSCs, cell passages, donor age, and their bioactivity profile differs, and hence the therapeutic benefits also vary. This distinction should be considered methodically when choosing the MSC source for specific clinical applications.

\section{CONCLUSION}

The study shows that WJ-MSCs are capable of protecting the endured islet mass along with the reversal of hyperglycaemia when injected into diabetic rats. These results suggests that MSC from Wharton's jelly could be a component of an innovative and successful cell-based regimen for diabetes treatment. An important lesson from intervention therapies in diabetes is that monotherapy often fails to provide a durable favourable outcome, particularly for reversal of the disease. WJ-MSC therapy could allow investigators to successfully manage diabetes with islet tissue protection, as well as regeneration and overall well being of the patient.

\section{REFERENCES}

1. Yang Zhao Wenyu Ye, Kristina S Boye, John H Holcombe, Jerry A Hall, Ralph Swindle. Prevalence of other diabetes-associated complications and comorbidities and its impact on health care charges among patients with diabetic neuropathy. Journal of Diabetes and its complications 2010:24, Issue 1, 9-19.

2. Liu X, Turban S, Carter R N et al. (2014) B-Cell-Specific Glucocorticoid Reactivation Attenuates Inflammatory B-Cell Destruction. Front Endocrinol (Lausanne) 5: 165.

3. Yoon JW, Jun HS. Cellular and molecular pathogenic mechanisms of insulin-dependent diabetes mellitus. AnnNYAcadSci. 2001 Apr; 928: 200-11.

4. Brownlee M. Biochemistry and molecular cell biology of diabetic complications. Nature 2001; 414: 813-820.

5. Mabed M1, Shahin M. Mesenchymal stem cell-based therapy for the treatment of type 1 diabetes mellitus. Curr Stem Cell Res Ther. 2012; 7: 179-190.

6. Fiorina P, Jurewicz M, Augello A, et al. Immunomodulatory function of bone marrow-derived mesenchymal stem cells in experimental autoimmune type 1 diabetes. J Immunol. 2009; 183: 993-1004.

7. Berman DM, Willman MA, Han D, Kleiner G, Kenyon NM, Cabrera O, Karl JA, Wiseman RW, O’Connor DH, Bartholomew AM, Kenyon NS. Mesenchymal stem cells enhance allogeneic islet engraftment in nonhuman primates. Diabetes. 2010.

8. Lu D, Chen B, Liang Z, et al. Comparison of bone marrow mesenchymal stem cells with bone marrow-derived mononuclear cells for treatment of diabetic critical limb ischemia and foot ulcer: a double-blind, randomized, controlled trial. Diabetes Res Clin Pract. 2011; 92: 26-36.

9. Jansen BJ, Gilissen C, Roelofs H, Schaap-Oziemlak A, Veltman JA, Raymakers RA, Jansen JH, Kögler G, Figdor CG, Torensma R, Adema GJ. Functional differences between mesenchymal stem cell populations are reflected by their transcriptome. Stem Cells Dev. 2010; 19(4): 481-90.

10. Sekiya I, Larson BL, Smith JR, Pochampally R, Cui J-G, Prockop DJ, Expansion of human adult stem cells from bone marrow stroma: 
conditions that maximize the yields of early progenitors and evaluate their quality, Stem Cells 2002; 20: 530-541.

11. Beane OS, Fonseca VC, Cooper LL, Koren G, Darling EM. Impact of aging on the regenerative properties of bone marrow-, muscle-, and adipose-derived mesenchymal stem/stromal cells. PLoS One. 2014 Dec 26; 9(12): el15963.

12. Kalaszczynska I, Ferdyn K. Wharton's jelly derived mesenchymal stem cells: future of regenerative medicine? Recent findings and clinical significance. BioMed Res Int. 2015; 2015: 430847.

13. Rydgren T, Vaarala O, Sandler S. Simvastatin protects against multiple low-dose streptozotocin-induced type 1 diabetes in CD-1 mice and recurrence of disease in nonobese diabetic mice. J Pharmacol Exp Ther. 2007 Oct; 323(1): 180-5.

14. Pal R, Hanwate M, Jan M, Totey S. Phenotypic and functional comparison of optimum culture conditions for upscaling of bone marrow-derived mesenchymal stem cells. Journal of Tissue Engineering and Regenerative Medicine 2009; 3(3): 163-174.

15. Singh GM, Danaei G, Farzadfar F, Stevens GA, Woodward M, Wormser DK, et al. The age-specific quantitative effects of metabolic risk factors on cardiovascular diseases and diabetes: a pooled analysis. PLoS. One. 2013; 8(7)e65174.

16. Abdi R, Fiorina P, Adra CN, Atkinson M, Sayegh MH.Immunomodulation by mesenchymal stem cells: a potential therapeutic strategy for type 1 diabetes. Diabetes 2008; 57: 1759-1767.

17. Ben-Ami E, Berrih-Aknin S, Miller A. Mesenchymal stem cells as an immunomodulatory therapeutic strategy for autoimmune diseases. Autoimmun Rev. 2011; 10: 410-415.
18. Jurewicz M, Yang S, Augello A, et al. Congenic mesenchymal stem cell therapy reverses hyperglycemia in experimental type 1 diabetes. Diabetes 2010; 59: 3139-3147.

19. Reddi AS, Kuppasani K, Ende N. Human umbilical cord blood as an emerging stem cell therapy for diabetes mellitus. Curr Stem Cell Res Ther. 2010; 5: 356-361.

20. Bárcia RN, Santos JM, Filipe M, Teixeira M, Martins JP, Almeida J, Água-Doce A, Almeida SCP, Varela A, Pohl S, Dittmar KEJ, Calado S, Simões SI, Gaspar MM, Cruz MEM, Lindenmaier W, Graça L, Cruz H, Cruz PE. What Makes Umbilical Cord Tissue-Derived Mesenchymal Stromal Cells Superior Immunomodulators When Compared to Bone Marrow Derived Mesenchymal Stromal Cells?Stem Cells International Volume 2015, Article ID 583984

21. Hass R, Kasper C, Böhm S, Jacobs R. Different populations and sources of human mesenchymal stem cells (MSC): A comparison of adult and neonatal tissue-derived MSC. Cell Commun Signal. 2011; 9: 12.

22. Marcus AJ, Woodbury D. Fetal stem cells from extra-embryonic tissues: Do not discard. J Cell Mol Med. 2008; 12: 730-742.

23. Chao LiGuojun WeiQun GuGang WenBaochang QiLiang XuShuqin Tao. Donor Age and Cell Passage Affect Osteogenic Ability of Rat Bone Marrow Mesenchymal Stem Cells Cell Biochemistry and Biophysics June 2015, Volume 72, Issue 2, pp 543-549.

24. Weiss ML, Anderson C, Medicetty S, et al, "Immune properties of human umbilical cord Wharton's jelly-derived cells", Stem Cells, vol. 26, no. 11, pp. 2865-2874, 2008. 